> Les dermatomyosites (DM) sont des maladies auto-immunes rares du groupe des myopathies inflammatoires idiopathiques, définies par une atteinte cutanée caractéristique. Elles peuvent survenir dans l'enfance, ou chez l'adulte. II existe des variations phénotypiques entre les DM concernant la présentation cutanéomusculaire (ex: amyopathique) mais aussi la présentation extra-cutanéomusculaire (ex: atteinte pulmonaire ou articulaire associée). Le caractère auto-immun de ces pathologies est souligné dans $60 \%$ des cas par la présence d'anticorps spécifique de myosite. Ces derniers sont associés à la présence de caractéristiques cliniques, histologiques, mais aussi pronostiques. Ils sont au nombre de cinq, les anti-Mi2, anti-Tifl- $\gamma$, antiNXP2, anti-MDA5 et anti-SAE. Les anti-Mi2 sont associées à une forme clinique cutanée classique, une atteinte musculaire souvent sévère au diagnostic et une bonne évolution sous traitement. Les deux suivants, fréquents chez l'enfant et l'adulte, sont associés à des formes récidivantes cutanées et sont fortement associés aux cancers chez l'adulte. Les anti-MDA5 sont les anticorps associés aux formes les plus systémiques avec une atteinte pulmonaire interstitielle rapidement progressive pouvant être très grave. Enfin, les anti-SAE n'ont été décrits que chez l'adulte, avec une atteinte classique. <

Les dermatomyosites (DM) sont des maladies autoimmunes rares du groupe des myopathies inflammatoires idiopathiques, définies par une atteinte cutanée caractéristique [1]. Elles peuvent survenir dans l'enfance [2], ou chez l'adulte. II existe des variations phénotypiques entre les DM concernant la présentation cutanéomusculaire (ex: amyopathique) mais aussi la présentation extra-cutanéomusculaire (ex: atteinte pulmonaire ou articulaire associée). À ce jour, le dia-

\section{Dermatomyosites \\ Nouveaux \\ anticorps, \\ nouvelle \\ classification}

Loïs Bolko ${ }^{1}$, Cyril Gitiaux², Yves Allenbach ${ }^{3}$

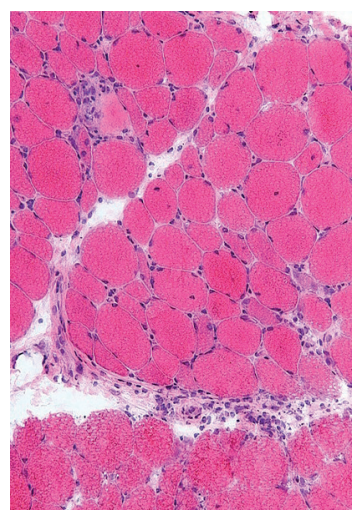
${ }^{1}$ Service de Rhumatologie Maison Blanche, CHU de Reims, Reims, France.
${ }^{2}$ Centre de référence des patho- logies neuromusculaires Paris Nord Est, service de neurophy- siologie clinique, Hôpital univer- sitaire Necker Enfants Malades, APHP.5, Université de Paris, France.
${ }^{3}$ Service de médecine interne et immunologie clinique, APHP Pitié-Salpêtrière, Paris, France. yves.allenbach@aphp.fr

gnostic de DM repose sur la présence de caractéristiques cliniques et histologiques, sans tenir compte des anticorps spécifiques de myosite (ASM), ces derniers permettant de définir des phénotypes homogènes de patients [3-4].

\section{Les anticorps spécifiques de myosite}

\section{Différents types d'anticorps}

Le groupe des myopathies inflammatoires idiopathiques (MII), ou myosites, peut être divisé (pour l'adulte) en quatre sous-groupes comprenant les DM, le syndrome des anti-synthétases (SAS), les myopathies nécrosantes auto-immunes (MNAI) et les myosites à inclusions (MI) [5]. Chaque sous-groupe présente des caractéristiques musculaires cliniques et anatomopathologiques propres ainsi qu'un phénotype extra-musculaire homogène. De même, chacun peut être associé à la présence d'ASM dont la cible antigénique dépend du groupe auquel il appartient. Parmi ces ASM, cinq ont été décrits pour les DM : les anticorps anti-Mi-2, anti-transcription intermediary factor $1 \gamma$ (TIFl $\gamma$ ), anti-nuclear matrix protein 2 (NXP2), anti-melanoma differentiation antigen 5 (MDA5) et anti-small ubiquitin-like modifier activating enzyme (SAE).

II existe sept anticorps associés au SAS, tous ciblant un ARN-t-synthétase. II s'agit des anti-Jo-1, ciblant un Histidyl-tRNA synthetases, les anti-PL-12 ou anti-Alanyl-tRNA synthetases, anti-PL-7 ou antiThreonyl-tRNA synthetases, anti-EJ ou anti-Glycyl-tRNA synthetases, 
anti-0) ou anti-Isoleucyl-tRNA synthetases, anti-KS ou anti-Asparaginyl-tRNA synthetases, anti-Zo ou anti-Phenylalanyl-tRNA synthetases, anti-Ha/YRS ou anti-Tyrosyl-tRNA synthetase.

Deux ASM sont associés au MNAI: les anti-3-hydroxy-3-methylglutaryl-coenzyme A reductase (HMGCR) et signal-recognition particle (SRP). Les anti-CNla sont associés aux myosites à inclusion.

\section{Historique, description et chronologie}

L'une des premières classifications et les premiers critères diagnostiques furent publiés par Peter et Bohan, en 1975 [6]. Fondée sur les caractéristiques clinico-histologiques, elle définit le diagnostic de myopathie inflammatoire et distingue les DM par la présence d'une atteinte cutanée caractéristique. Depuis, les ASM ont été progressivement décrits. Le premier fut l'anticorps anti-Mi2 en 1976 [7]. Puis, la majorité des anticorps ciblant une aminoacyl-ARN-t synthétase a été découverte entre 1980 (avec celle de l'anticorps Jol, chef de file de ce groupe) et 1991. Love et al ont évalué, en 1991, l'apport de ces nouveaux anticorps dans une cohorte de 212 patients suivis pour des MII [8]. Ils ont montré pour la première fois que ces ASM permettaient de regrouper les patients en groupes homogènes clinico-biologiques, mais aussi pronostiques.

Puis, en 2003, la conférence ENMC (European Neuromuscular Center) précise les critères histologiques de la biopsie musculaire pour le diagnostic de DM [4] avec notamment l'atrophie périfasciculaire de la DM ou encore la présence d'une vasculopathie, mais les ASM ne sont pas intégrés dans la classification. Au décours, six autres anticorps ont été décrits, dont quatre associés aux DM. Dans le courant des années 2000 de nombreux travaux décrivant des séries de cas permettront d'établir que cinq ASM sont spécifiques de la DM. Malgré ces avancées, la dernière classification de l'American College of Rheumatology et de l'European League Against Rheumatism (ACR/EULAR) de 2017 ne les ont pas inclus comme critères diagnostiques de myosite ni de classification pour le groupe DM [3]. Une étude récente utilisant une analyse non supervisée de regroupement hiérarchique a montré que les ASM étaient associés avec des phénotypes cliniques correspondant aux quatre groupes actuels de MII [5].

\section{Méthode de détection des Ac}

L'immunoprécipitation est la méthode de référence de détection des ASM. Cette technique n'est pas réalisable en pratique courante. La recherche d'anti-nucléaires pour le dépistage des ASM n'est pas suffisamment sensible. Afin d'améliorer la détection de ces ASM, plusieurs laboratoires ont développé des tests de détection multiple, (test immunodiagnostique par exemple DOT-Myosite), permettant d'évaluer de manière rapide et simultanée la présence de plusieurs ASM à partir du sérum de patient [9]. Néanmoins, la fiabilité n'est pas parfaite et nécessite une interprétation précautionneuse avec les données clinico-biologiques, compte tenu de la présence de faux positifs.

\section{Les ASM dans les DM}

Chaque ASM spécifique des DM est associé à des particularités cliniques musculaires, cutanées et/ou systémiques; biologiques, histologiques mais aussi pronostiques. Dans les DM et les formes juvéniles (JDM), un autoanticorps est identifié dans environ $60 \%$ des cas. Dans l'ensemble les caractéristiques phénotypiques associées au différents ASM des DM de ses anticorps sont similaires entre l'adulte et l'enfant, à l'exception de l'association au cancer. Néanmoins, il existe des différences de prévalence. Les anticorps anti-TIFl $\gamma$, NXP2 et MDA5 sont les plus fréquents dans les JDM, avec la répartition suivante 18 \%-15\%-6\% [2]. Chez l'adulte, les ASM les plus représentés sont les anti-TIFl $\gamma(38-41 \%)$, Mi-2 (2-38 \%) et NXP2 (14-25\%) chez le sujet caucasien [1]. À l'inverse, les anti-SAE sont les anticorps les moins prévalents, et sont détectés chez l'adulte presque exclusivement dans 1-10\% des cas, plutôt chez le sujet caucasien (Tableau I).

\section{Anti-Mi-2}

Ce fut le premier ASM décrit en 1976 [7]. La protéine Mi2 est une sous-unité du complexe Mi-2/nucleosome remodeling-deacetylase, qui régule la transcription des gènes. Les patients avec anticorps anti-Mi2 ont une atteinte cutanée caractéristique, avec un érythème dans les zones photo-exposées, un érythème liliacé des paupières et la présence de papule de Gottron [10]. L'atteinte musculaire est relativement sévère avec un taux élevé de créatine phosphokinase (CPK) chez l'adulte et l'enfant [11] et avec une inflammation importante à la biopsie musculaire [12].

II y a peu d'atteinte extra-musculaire et cutanée dans ce sous-groupe, peu d'association avec les cancers hormis peut-être pour les anticorps Mi2 ciblant la partie $\mathrm{N}$-terminale du fragment Mi2- $\beta$ chez l'adulte] [10]. La réponse au traitement et le pronostic sont plutôt bons, avec des rechutes néanmoins possibles à long terme.

\section{Anti-TIFl $\gamma$}

Cet ASM a été initialement découvert en 2006 par une équipe américaine [13]. Cette protéine est impliquée dans la croissance et la différenciation cellulaire mais aussi dans la carcinogénèse [14]. L'atteinte cutanée est au premier plan chez ces patients, avec des récidives plus fréquentes [15]. Outre l'érythème typique de DM, les patients ont des lésions caractéristiques «psoriasiformes », des papules de Gottron hyperkératosiques ou encore des lésions hypo-pigmentées et télangiectasiques ("red on white patches") [14-15]. L'atteinte musculaire n'est habituellement pas au premier plan, avec des formes dites «amyopathiques » fréquentes. La biopsie musculaire retrouve une atteinte sévère quand elle est présente, avec des signes de vasculopathie et une dysfonction mitochondriale plus importante [12]. De plus, il a été décrit la présence de 


\begin{tabular}{|c|c|c|c|c|c|}
\hline & SAE & Mi2 & NXP2 & TIFl $\gamma$ & MDA5 \\
\hline $\begin{array}{l}\text { Atteinte } \\
\text { cutanée }\end{array}$ & $\begin{array}{l}\text { Sévère } \\
\text { Atteinte typique } \\
\text { Erythème violacé } \\
\text { inconstant }\end{array}$ & Erythème typique & $\begin{array}{l}\text { Calcinose } \\
\text { Oedème sous cutané }\end{array}$ & $\begin{array}{l}\text { Sévère } \\
\text { Papules de Gottron } \\
\text { hyperkératosique } \\
\text { Lésions } \\
\text { psoriasiformes } \\
\text { Patch 'red on white' }\end{array}$ & $\begin{array}{l}\text { Mains de mécanicien } \\
\text { Ulcère cutané } \\
\text { Papule palmaire }\end{array}$ \\
\hline $\begin{array}{l}\text { Atteinte } \\
\text { musculaire }\end{array}$ & Dysphagie & $\begin{array}{l}\text { Atteinte sévère } \\
\text { Taux élevé de CPK }\end{array}$ & $\begin{array}{l}\text { Atteinte sévère } \\
\text { Myalgie } \\
\text { Dysphagie }\end{array}$ & $\begin{array}{l}\text { Atteinte modérée } \\
\text { Taux faible de CPK } \\
\text { «DM } \\
\text { amyopathique» } \\
\text { Dysphagie }\end{array}$ & $\begin{array}{l}\text { «DM } \\
\text { amyopathique }\end{array}$ \\
\hline $\begin{array}{l}\text { Atteintes } \\
\text { extra-cutanéo- } \\
\text { musculaires }\end{array}$ & $\begin{array}{l}\text { PID : Peu fréquente } \\
\text { Arthrite : Peu } \\
\text { fréquente } \\
\text { Raynaud : Peu } \\
\text { fréquent }\end{array}$ & $\begin{array}{l}\text { PID : Peu fréquente } \\
\text { Arthrite: Peu } \\
\text { fréquente Raynaud : } \\
\text { Peu fréquente }\end{array}$ & $\begin{array}{l}\text { PID : Peu fréquente } \\
\text { Arthrite: Peu } \\
\text { fréquente } \\
\text { Raynaud: Peu } \\
\text { fréquente }\end{array}$ & $\begin{array}{l}\text { PID : Peu fréquente } \\
\text { Arthrite : Peu } \\
\text { fréquente } \\
\text { Raynaud : Peu } \\
\text { fréquente }\end{array}$ & $\begin{array}{l}\text { PID sévère } \\
\text { Arthrite : fréquente } \\
\text { Raynaud : fréquent } \\
\text { Fièvre }\end{array}$ \\
\hline $\begin{array}{l}\text { Association } \\
\text { au cancer }\end{array}$ & $0-20 \%$ & $0-20 \%$ & $10-35 \%$ & $35-80 \%$ & 0 \\
\hline $\begin{array}{l}\text { Histologie } \\
\text { musculaire }\end{array}$ & $\begin{array}{l}\text { Lésions peu sévères } \\
\text { Atteinte classique }\end{array}$ & $\begin{array}{l}\text { Lésions sévères } \\
\text { Lésion nécrotique }\end{array}$ & $\begin{array}{l}\text { Lésions sévères } \\
\text { Vasculopathie }\end{array}$ & $\begin{array}{l}\text { Lésions sévères } \\
\text { Vasculopathie }\end{array}$ & $\begin{array}{l}\text { Lésions peu sévères } \\
\text { Atteinte focale }\end{array}$ \\
\hline
\end{tabular}

Tableau I. Caractéristiques phénotypiques des dermatomyosites associées aux différents anticorps spécifiques de myosite. PID : pneumopathie interstitielle diffuse.

fibres vacuolées, sans vacuoles bordées. Chez l'adulte, la présence de cet anticorps est fortement associée au cancer. En effet, une métaanalyse de six études retrouve un risque de cancer augmenté avec un odds ratio de 27.26 (IC95 6.59-112.82) [16]. Les cancers sont diagnostiqués majoritairement dans l'année précédant ou suivant le diagnostic de DM, mais ne sont pas associés à un sous-type particulier de cancer [17]. De plus, le sous-type IgG2 de l'anticorps anti TIFl $\gamma$ est fortement associé aux cancers [18].

Dans les JDM, les anticorps anti-Tifl $\gamma$ sont identifiés dans $18 \%-30 \%$ des cas $[2,19]$. Cet anticorps semble être associé à des formes plus chroniques ou récidivantes nécessitant l'adjonction plus fréquente d'une deuxième ligne de traitement [20]. Les atteintes cliniques semblent hétérogènes avec des JDM « classiques » peu sévères et des formes graves pouvant être similaires à celles décrites pour les antiNXP2 [2].

\section{Anti-NXP2}

Les Ac anti-NXP2 ont été découverts en 1997 dans les JDM. La protéine NXP2 est associée à l'enzyme SAE, utile à la répression de la transcription.
Les anticorps anti-NXP2 sont identifiés dans environ $15 \%-20 \%$ des JDM et sont généralement trouvés chez des enfants plus jeunes par rapport aux autres ASM. Ils sont associés à une atteinte musculaire plus sévère au diagnostic avec une tendance à présenter davantage de calcinose musculaire au cours de l'évolution $[2,19]$. Cependant le lien entre la présence de la calcinose et cet anticorps reste à confirmer car il est aussi probable que la sévérité de l'atteinte musculaire, le délai dans la prise en charge et une maladie chronique soient aussi des facteurs favorisants de calcinose indépendamment de la présence de l'anticorps. La physiopathologie de la calcinose reste non élucidée, échappe souvent à toute thérapeutique et retentit sur la qualité de vie des patients [21]. Sur le plan histologique, la gravité de l'atteinte associée à ces anticorps peut être variable [22] mais il existe une forme particulièrement grave de JDM avec AcNXP2 qu'il faut reconnaitre rapidement pour mettre en place une thérapeutique adaptée, associant une atteinte musculaire sévère incluant les muscles respiratoires et 
bulbaires, une atteinte digestive, une atteinte cutanée avec les signes classiques de DM associée à des œdèmes diffus [23]. Dans ce contexte, la biopsie musculaire montre une atteinte ischémique avec une atteinte majeure de la micro-vascularisation musculaire. L'association de la présence d'anticorps anti-NXP2 à une atteinte ischémique musculaire sont des biomarqueurs complémentaires de sévérité clinique et de mauvais pronostic devant conduire à l'instauration rapide d'une seconde ligne de traitement immunomodulateur [23].

Chez l'adulte, il existe une incidence plus importante d'œdème souscutané [24]. L'atteinte musculaire est caractérisée par des signes de vasculopathie, avec une raréfaction des capillaires ainsi qu'un œdème musculaire fréquent [12]. Dans ce sous-groupe, il existe aussi une incidence plus importante de cancer chez l'adulte, notamment de sexe masculin [17], mais le risque est plus faible qu'en présence des antiTIFl $\gamma$.

\section{Anti-MDA5}

Cet anticorps a été décrit en 2005 sur une cohorte de patients japonais suivis pour des dermatomyosites amyopathiques [25]. MDA5 est une protéine de la famille des retinoic acid-inducible gene I (RIG-I-like receptors), ayant un rôle dans l'immunité antivirale et notamment la voie de l'interféron. Les DM associées à cet Ac sont les DM avec le plus d'atteintes systémiques et le pronostic le plus sombre en raison d'une atteinte pulmonaire dans plus de $80 \%$ des cas. Cette dernière peut engager le pronostic vital. Sur le plan cutané, l'atteinte se distingue par la présence d'ulcérations cutanées souvent sévères, ainsi que la présence de mains de mécanicien [26]. L'atteinte musculaire est habituellement modeste voire absente avec un taux de CPK souvent normal. Les données de la biopsie musculaire retrouvent plus rarement les signes classiques de DM comme l'atrophie péri-fasciculaire ou l'expression de HLA-1 [27]. Parmi les autres signes cliniques, les patients présentent une atteinte articulaire inflammatoire avec arthralgie et arthrite, ainsi qu'une fièvre constitutionnelle. Mais l'atteinte systémique qui grève le pronostic dans ce sous-groupe est la présence d'une atteinte pulmonaire interstitielle rapidement progressive [28].

Dans les JDM, les anticorps anti-MDA5 sont les troisièmes anticorps en fréquence identifiés dans les myopathies inflammatoires de l'enfant (6-7\%) [2,19]. Les myosites pédiatriques associées aux anticorps anti-MDA5 représentent une entité clinique assez homogène en cours de définition. En effet, tout comme chez l'adulte, elles sont caractérisées essentiellement par des signes extra-musculaires au premier plan. Même si l'atteinte pulmonaire est moins fréquente ( $46 \%$ ) elle peut être aussi sévère (pneumopathie interstitielle progressive qui constitue la gravité de cette maladie). L'atteinte musculaire est le plus souvent absente (amyopathique) ou modérée et l'atteinte cutanée est caractéristique avec ulcérations plus fréquentes (69\%). Les arthrites (100\%) sont très fréquentes et on peut noter la présence des signes de la série lupique (46\%) ou des abcès aseptiques (17\%) [2, Melki et al soumis]. L'atteinte musculaire sur le plan histologique est aussi significativement moins sévère comparativement aux autres auto-anticorps avec un infiltrat inflammatoire souvent modéré et l'absence d'atteinte de la microvascularisation.

\section{Anti-SAE}

Cet anticorps est le dernier ASM spécifique de DM décrit en 2007 [29]. Cette protéine facilite la sumoylation, une modification post-translationnelle de nombreuses protéines. L'atteinte cutanée est souvent révélatrice, typique avec dans de rares cas des atteintes cutanées spécifiques avec un rash cutané violacé [30] (Tableau I). Ces patients présentent aussi des symptômes systémiques avec une fièvre inexpliquée, une altération de l'état général. L'atteinte musculaire n'est habituellement pas au premier plan, avec des CPK dans les normes ou faiblement augmentés. Des troubles de la déglutition ont été décrits ainsi que des cancers associés [30].

\section{Application en pratique clinique}

\section{Rôle diagnostique}

Le rôle diagnostique du dosage de ces anticorps est donc évident, permettant en premier lieu de distinguer les myopathies auto-immunes des myopathies non inflammatoires. Les ASM sont spécifiques et ne sont pas retrouvés dans d'autres pathologies musculaires pédiatriques ou de l'adulte. Leur dosage doit être réalisé systématiquement devant tout déficit musculaire aigu/subaigu, car leur identification modifie drastiquement la prise en charge thérapeutique initiale. À l'inverse, l'absence d'ASM dans un contexte clinique concordant n'élimine pas ce diagnostic et dans ce contexte la biopsie musculaire reste utile pour confirmer le diagnostic avant de débuter des traitements immunomodulateurs au long cours. En effet, la mise en évidence de ces auto-anticorps a permis de questionner la place de la biopsie musculaire dans la stratégie diagnostique. Cependant, des études récentes ont montré que l'analyse immuno-pathologique permet de déterminer précisément le sous-type de myopathie inflammatoire et participe à l'évaluation de la sévérité et du pronostic.

Le deuxième rôle des ASM est le diagnostic différentiel dans les atteintes cutanées isolées des formes amyopathiques. La présence des lésions cutanées pathognomoniques n'est pas toujours présente et les lésions peuvent être discrètes; se pose alors la question du diagnostic différentiel avec le lupus érythémateux systémique, le psoriasis ou encore des viroses comme le parvovirus B19 ou des carences rares en zinc. La présence d'un ASM dans ce contexte permet d'orienter le diagnostic et la prise en charge thérapeutique qui sera différente.

Bien classifier ces patients permet enfin la constitution de groupes homogènes de patients pour une meilleure compréhension de la physiopathologie de la maladie, et de préciser le pronostic de la DM. 


\section{Rôle pronostique}

L'utilité de ces ASM est aussi pronostique. La différence majeure entre les DM et les JDM est l'association aux cancers. Chez l'adulte, la présence des anticorps anti-TIFl $\gamma$ et NXP2 doivent faire rechercher un cancer associé en répétant le bilan à un et deux ans du diagnostic initial.

La présence d'anticorps anti-MDA5 permet de monitorer la fonction pulmonaire de manière rapprochée avec une démarche thérapeutique plus agressive compte tenu du pronostic plus sévère dans ce sousgroupe, dû à l'atteinte pulmonaire rapidement progressive. La présence d'anti-TIFl $\gamma$ sera associée à des rechutes plus fréquentes chez l'enfant. La présence de signes systémiques associés à la positivité d'un anticorps anti-NXP2 devra conduire à une thérapeutique rapide dans les formes juvéniles.

II n'existe actuellement pas encore de stratégie thérapeutique validée ajustée à la présence d'un anticorps spécifique.

\section{Conclusion}

Ces anticorps ont donc un rôle diagnostique et pronostique important, permettant de définir des sous-groupes homogènes de patients. En 2019, une réunion sous l'égide de l'European Neuromuscular Center dédiée à la révision des critères diagnostiques des DM a décidé d'inclure les ASM dans les critères diagnostiques des MII. Les avancées de la compréhension physiopathologique pourraient permettre dans l'avenir de définir des stratégies thérapeutiques adaptées aux différents anticorps.

\section{SUMMARY}

Dermatomyositis: new antibody, new classification

Dermatomyositis are rare chronic auto-immune diseases characterized by cutaneous involvement. Diagnosis could be made in childhood or in aldult. There are some different clinical and histological presentation associated with different myositis specific antibody. There are five dermatomyositis specific autoantibodies, anti-Mi2, anti-Tifl- $\gamma$, antiNXP2, anti-MDA5, and anti-SAE. Anti-Mi2 are associated with "classical form" of DM with cutaneous and muscular involvement. Anti-Tifl $\gamma$ and anti-NXP2 are found in juvenile and adult dermatomyositis, and are associated with recurrent diseases with cutaneous involvement at the forefront. In adults, they are associated with cancer. Anti-MDA5 antibodies are associated with a systemic involvement and an interstitial lung disease. Finally, anti-SAE have been found only in adults, with a classic form. $\diamond$

\section{LIENS D’INTÉRÊT}

Les auteurs déclarent n'avoir aucun lien d'intérêt concernant les données publiées dans cet article.

\section{RÉFÉRENCES}

1. Wolstencroft PW, Fiorentino DF. Dermatomyositis clinical and pathological phenotypes associated with myositis-specific autoantibodies. Curr Rheumatol Rep $2018 ; 20: 28$.

2. Tansley SL, Simou S, Shaddick G, et al. Autoantibodies in juvenile-onset myositis: their diagnostic value and associated clinical phenotype in a large UK cohort. J Autoimmun 2017 ; 84 : 55-64.
3. Lundberg $I E$, Tjärnlund A, Bottai M, et al. 2017 European league against rheumatism/American college of rheumatology classification criteria for adult and juvenile idiopathic inflammatory myopathies and their major subgroups. Arthritis Rheum (Hoboken, NJ) $2017 ; 69: 2271-82$.

4. Hoogendijk JE, Amato AA, Lecky BR, et al. $119^{\text {th }}$ ENMC international workshop: trial design in adult idiopathic inflammatory myopathies, with the exception of inclusion body myositis, 10-12 October 2003, Naarden, The Netherlands. Neuromuscul Disord 2004 ; 14 : 337-45.

5. Mariampillai K, Granger B, Amelin D, et al. Development of a new classification system for idiopathic inflammatory myopathies based on clinical manifestations and myositis-specific autoantibodies. JAMA Neurol $2018 ; 75: 1528-37$.

6. Bohan A, Peter JB. Polymyositis and dermatomyositis (first of two parts). N Engl J Med 1975 ; 292 : 344-7.

7. Reichlin M, Mattioli M. Description of a serological reaction characteristic of polymyositis. Clin Immunol Immunopathol 1976; 5 : 12-20.

8. Love LA, Leff RL, Fraser DD, et al. A new approach to the classification of idiopathic inflammatory myopathy: myositis-specific autoantibodies define useful homogeneous patient groups. Medicine (Baltimore) 1991; 70 : 360-74.

9. Damoiseaux J, Vulsteke JB, Tseng CW, et al. Autoantibodies in idiopathic inflammatory myopathies: clinical associations and laboratory evaluation by mono- and multispecific immunoassays. Autoimmun Rev 2019 ; 18 : 293-305.

10. Hengstman GJD, Vree Egberts WTM, Seelig HP, et al. Clinical characteristics of patients with myositis and autoantibodies to different fragments of the Mi-2 beta antigen. Ann Rheum Dis 2006 ; 65 : 242-5.

11. Landon-Cardinal 0 , Monseau G, Schoindre Y, et al. Anti-Mi2 dermatomyositis revisited: pure DM phenotype with muscle fiber necrosis and high risk of malignancy [abstract]. Arthritis Rheumatol 2016; 68 (suppl 10). Accessed July 20, 2017.

12. Pinal-Fernandez I, Casciola-Rosen LA, Christopher-Stine L, et al. The prevalence of individual histopathologic features varies according to autoantibody status in muscle biopsies from patients with dermatomyositis. J Rheumatol 2015 ; 42 : 1448-54.

13. Targoff IN, Mamyrova G, Trieu EP, et al. A novel autoantibody to a $155-\mathrm{kd}$ protein is associated with dermatomyositis. Arthritis Rheum 2006 ; 54 : 3682-9.

14. Fujimoto M, Hamaguchi Y, Kaji K, et al. Myositis-specific anti-155/140 autoantibodies target transcription intermediary factor 1 family proteins. Arthritis Rheum 2012; 64 : 513-22.

15. Fiorentino DF, Kuo K, Chung L, et al. Distinctive cutaneous and systemic features associated with antitranscriptional intermediary factor- $1 \gamma$ antibodies in adults with dermatomyositis. J Am Acad Dermatol 2015 ; 72 : 449-55.

16. Trallero-Araguás $\varepsilon$, Rodrigo-Pendás JÁ, Selva-0'Callaghan A, et al. Usefulness of anti-p155 autoantibody for diagnosing cancer-associated dermatomyositis: a systematic review and meta-analysis. Arthritis Rheum $2012 ; 64: 523-32$

17. Fiorentino DF, Chung LS, Christopher-Stine L, Zaba L, Li S, Mammen AL, et al. Most patients with cancer-associated dermatomyositis have antibodies to nuclear matrix protein NXP-2 or transcription intermediary factor $1 \gamma$. Arthritis Rheum $2013 ; 65$ : 2954-62.

18. Aussy A, Fréret $M$, Gallay L, et al. The IgG2 isotype of anti-transcription intermediary factor 1-gamma autoantibodies is a biomarker of mortality in adult dermatomyositis. Arthritis Rheumatol (Hoboken NJ) 2019; $71: 1360$ 70.

19. McHugh NJ, Tansley SL. Autoantibodies in myositis. Nat Rev Rheumatol $2018 ; 14: 290-302$

20. Gunawardena H, Wedderburn LR, North, J, et al. Clinical associations of autoantibodies to a p155/140 kDa doublet protein in juvenile dermatomyositis. Rheumatology (Oxford) $2008 ; 47: 324-8$.

21. Orandi AB, Dharnidharka VR, Al-Hammadi N, et al. Clinical phenotypes and biologic treatment use in juvenile dermatomyositis-associated calcinosis. Pediatr Rheumatol Online J $2018 ; 16: 84$.

22. Yasin SA, Schutz PW, Deakin CT, et al. Histological heterogeneity in a large clinical cohort of juvenile idiopathic inflammatory myopathy: analysis by myositis autoantibody and pathological features. Neuropathol Appl Neurobiol 2019 ; 45 : 495-512.

23. Aouizerate J, De Antonio M, Bader-Meunier B, et al. Muscle ischaemia associated with NXP2 autoantibodies: a severe subtype of juvenile dermatomyositis. Rheumatology (Oxford) 2018 ; 57 : 873-9. 


\section{RÉFÉRENCES}

24. Albayda J, Pinal-Fernandez I, Huang W, et al. Antinuclear matrix protein 2 autoantibodies and edema, muscle disease, and malignancy risk in dermatomyositis patients. Arthritis Care Res (Hoboken) $2017 ; 69: 1771-6$.

25. Sato S, Hirakata M, Kuwana M, et al. Autoantibodies to a 140-kd polypeptide, CADM-140, in Japanese patients with clinically amyopathic dermatomyositis. Arthritis Rheum $2005 ; 52$ : 1571-6.

26. Hall JC, Casciola-Rosen L, Samedy LA, et al. anti-Mda5-associated dermatomyositis: expanding the clinical spectrum. Arthritis Care Res (Hoboken) $2013 ; 65$ : 1307-15.

27. Allenbach $Y$, Leroux $G$, Suárez-Calvet $X$, et al. Dermatomyositis with or without anti-melanoma differentiation-associated gene 5 antibodies: common interferon signature but distinct NOS2 expression. Am J Pathol 2016; 186: 691-700

28. Moghadam-Kia S, Oddis CV, Sato S, et al. Anti-melanoma differentiation-associated gene 5 is associated with rapidly progressive lung disease and poor survival in US patients with amyopathic and myopathic dermatomyositis. Arthritis Care Res (Hoboken) 2016 ; $68: 689-94$.
29. Betteridge Z, Gunawardena H, North J, et al. Identification of a novel autoantibody directed against small ubiquitin-like modifier activating enzyme in dermatomyositis. Arthritis Rheum 2007 ; $56: 3132-7$.

30. Ge Y, Lu X, Shu X, et al. Clinical characteristics of anti-SAE antibodies in Chinese patients with dermatomyositis in comparison with different patient cohorts. Sci Rep [Internet]. 15 mars 2017 [cité 2 juin 2019]; 7. Disponible sur: https://www.ncbi.nlm.nih.gov/pmc/articles/PMC5428032/

\section{www.myobase.org}

Catalogue en ligne disponible gratuitement sur Internet publié par l'AFM-Téléthon.

Retrouvez facilement toutes les références bibliographiques sur les maladies neuromusculaires, les situations de handicap qu'elles génèrent et leurs aspects psychologiques.

\section{Myobase donne un} accès libre à $75 \%$ du fonds documentaire collecté depuis 1990, représentant plus de 40000 références spécifiques du domaine des maladies neuromusculaires.

$>$ articles de la littérature biomédicale et psycho-sociale

\section{$>$ livres, thèses}

$>$ guides d'associations et rapports institutionnels d'agences internationales

\section{$>$ brèves en français,} synthèses des articles médico-scientifiques internationaux les plus pertinents

\section{$>$ publications}

AFM-Téléthon destinées aux professionnels de santé ou aux personnes atteintes de maladie neuromusculaire et à leur entourage

\section{UN OUTIL ERGONOMIQUE, UNE INTERFACE BILINGUE}

- Laissez-vous guider par les tutoriels

- Lancez une recherche et affinez votre sélection grâce aux filtres

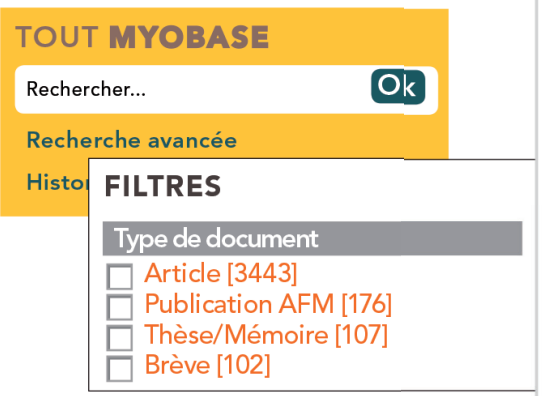

\section{- PUBLICATIONS AFM-Téléthon}

\section{- BRÈVES}

- DOCUMENTS DE SYNTHÈSE

INSTITUT DES BIOTHÉRAPIES PUBLICATIONS

- Partagez les résultats de votre recherche

\section{UN ACCÈS facile et simple}

Rechercher avec des opérateurs :

- guillemets pour une expression "maladie de pompe"

- + pour signifier ET, et retrouver tous les documents contenant les deux mots "fauteuil +électrique"

- - pour signifier NON et enlever le mot de la recherche:

"autonomie -établissement"
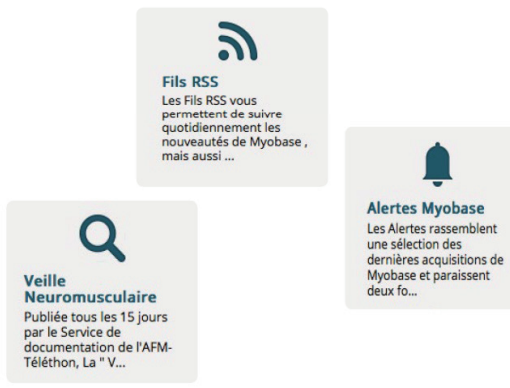

- Cliquez sur l'onglet

thématique qui vous convient (haut de la page d'accueil)

- Créez vos alertes personnalisées en ouvrant un compte personnel

- Téléchargez la Veille Neuromusculaire

- Abonnez-vous aux flux RSS 